\title{
Risk of traffic accidents in patients with sleep-disordered breath- ing: reduction with nasal CPAP
}

\author{
W. Cassel, T. Ploch, C. Becker, D. Dugnus, J.H. Peter, P. von Wichert
}

Risk of traffic accidents in patients with sleep-disordered breathing: reduction with nasal CPAP. W. Cassel, T. Ploch, C. Becker, D. Dugnus, J.H. Peter, P. von Wichert. (C)ERS Journals Ltd 1996.

ABSTRACT: Sleepiness whilst driving constitutes a road safety risk. Sleep-related breathing disorders are the most frequent medical cause of daytime sleepiness, and untreated patients with this condition have been shown to be at a higher risk of having accidents while driving. This study addressed the question of the extent to which treatment of sleep-disordered breathing by nasal continuous positive airway pressure (nCPAP) is related to changes in patient's accident risk.

Seventy eight male patients requiring treatment of sleep-related breathing disorders with nCPAP were enrolled in the study. The protocol included a questionnaire dealing with alertness-related problems while driving, an $80 \mathrm{~min}$ vigilance test, and the Multiple Sleep Latency Test. These baseline evaluations were repeated after 1 year of treatment with nCPAP.

Fifty nine patients completed the study. The accident rate was significantly decreased from 0.8 per $100,000 \mathrm{~km}$ (untreated) to 0.15 per $100,000 \mathrm{~km}$ with nCPAP treatment. Variables that were considered to be likely to increase accident risk (sleeping spells, fatigue, vigilance test reaction time, daytime sleep latency) also improved with treatment.

We conclude that treatment of sleep-disordered breathing by nasal continuous positive airway pressure is related to reduction in patient motor vehicle accident rates, probably due to the reversal of excessive daytime sleepiness.

Eur Respir J., 1996, 9, 2606-2611.

Sleepiness is being recognized increasingly as a possible traffic hazard [1, 2]. A recent study [3] evaluating all highway accidents with fatalities on Bavarian highways in 1991 concluded that falling asleep at the wheel was responsible for $24 \%$ of fatal accidents, and thus represents the most frequent and important factor contributing to death on Bavarian highways. In 1988, the Association of Professional Sleep Societies' "Committee on Catastrophes, Sleep and Public Policy" concluded that sleep and sleep-related factors appear to be involved in widely disparate types of disasters, from car accidents to nuclear plant catastrophes [4].

For the United States of America, LEGER [5] estimates the total cost of sleepiness-related accidents (motor-vehicle, work-related, home-based and public accidents) in 1988 to have been between 43 (lower estimate) and 56 billion (upper estimate) US dollars.

Several studies [6-12] have shown an elevated risk for traffic accidents in patients with sleep-related breathing disorders (SRBD). Although for a given amount of sleep-disordered breathing (as measured by polysomnography), not all patients will present with a similar level of impaired alertness, there is little doubt that SRBD represent a risk factor for accidents [13]. There have so far been only a few studies addressing the effects of treatment of SRBD on the patient's risk for traffic accidents. Findley et al. [14] and HaraldSSON et al. [15] have shown improved performance in driving simulator tests after treatment with nasal continuous positive airway
Klinikum der Philipps-Universität, Zentrum für Innere Medizin, Abteilung Poliklinik, Schlafmedizinisches Labor, Marburg, Germany.

Correspondence: W. Cassel Klinikum der Philipps-Universität Zentrum für Innere Medizin Abteilung Poliklinik Schlafmedizinisches Labor D-35033 Marburg Germany

\section{Keywords: Accidents}

nasal continuous positive airway pressure sleep-disordered breathing sleepiness

Received: February 131996 Accepted after revision August 201996

This study is part of the research project 2.9107: "Sleep related breathing disorders and traffic security", carried out on behalf of the "Bundesanstalt für Straßenwesen". pressure (nCPAP) and uvulopalatopharyngoplasty (UPPP) respectively.

Nasal CPAP, introduced by SULLIVAN in the early 1980s [16], is the most common treatment of SRBD, and is also viewed as the most effective treatment in most cases [17, 18]. MinEmuRA et al. [19] followed-up 14 patients with obstructive sleep apnoea for an average of 11 months after nCPAP was started, and observed no accidents in this period compared to $42 \%$ of these patients with accidents in the three preceding years.

The aim of this study was to determine the rate of traffic accidents in patients with sleep-related breathing disorders, before and after one year of treatment with nCPAP. In addition, changes in daytime vigilance and daytime sleepiness, as well as changes in fatigue-related problems while driving were measured before and after treatment in this patient group.

\section{Methods}

\section{Subjects}

Of all the male patients referred to the out-patient department of the Sleep Disorders Center at the University Hospital Marburg $(n=730)$ between January 1st 1992 and June 30th 1993, 78 were included in this study. Patients were included based on the following criteria: Caucasian; aged 25-65 yrs; possession of a driver's license, willingness to participate; presence of excessive 
daytime sleepiness (self-reported); and an indication for nCPAP treatment according to MESAM recordings, polysomnography and the patients individual risk.

Exclusion criteria were: chronic intake of sedatives; a diagnosis of narcolepsy; periodic limb movements; lung diseases; other chronic medical illnesses; known alcohol and drug abuse.

\section{Procedures}

All patients were initially referred to the out-patient department by their medical practitioner. Prior to all other procedures, a questionnaire dealing with alertnessrelated problems while driving was completed by each subject. As a part of the standard testing schedule, another questionnaire, including items on common symptoms of sleep apnoea, quality of life, and sleep habits, was also completed. A complete physical examination was performed and full medical history was recorded. MESAM IV [20-22] recordings were then performed on all patients and, according to their results, the necessity for full polysomnographic recordings in the sleep laboratory was subsequently determined.

An initial polysomnographic sleep study confirming the presence of SRBD and verifying the indication for nCPAP was undertaken. The following parameters were recorded continuously on chart recorders (ED-16 and UD-8; Madaus Schwarzer, Munich, Germany): two electroencephalograph (EEG) leads (C3/A2, C4/A1); two electro-oculograph (EOG) leads; one electromyograph (EMG) lead (chin); thoracic and abdominal movements by inductive plethysmography (Respitrace; Ambulatory Monitoring, Ardsley, NY, USA); oronasal airflow by thermistors; arterial oxygen saturation $\left(\mathrm{Sa}_{\mathrm{a}} \mathrm{O}_{2}\right)$ by means of finger oximetry (Nellcor, Hayward, CA, USA; 1channel electrocardiography (ECG).

In addition, a standard Multiple Sleep Latency Test (MSLT) [23] was performed on the day following the sleep study, using the montage from the preceding sleep study, except for all breathing parameters. During the MSLT, between the first and second sleep latency test, a four choice reaction time test, with a duration of approximately $80 \mathrm{~min}$, was performed.

Following this series of daytime tests, each patient spent another night in the sleep laboratory undergoing a pressure determination study for nCPAP treatment, during which the above-mentioned parameters, with the exception of EEG, were measured. Pressure was adjusted individually to prevent apnoeas, hypopnoeas and snoring. During a third night in the sleep laboratory, the accuracy of the defined nCPAP pressure was checked and corrected, if required.

Patients were encouraged to contact the sleep laboratory staff following discharge if any problems with nCPAP treatment arose. The patients' referring physicians were informed about the nCPAP treatment and encouraged to contact the sleep laboratory if they had additional questions concerning the treatment or if patients reported problems to them. Six months after treatment was initiated, an ambulatory MESAM IV recording was performed by the out-patient department of the sleep disorders unit as a further measure of the efficacy of the nCPAP therapy.

After 1 year of treatment, the accuracy and efficacy of the nCPAP therapy was formally retested in the sleep laboratory. The daytime testing schedule of the initial evaluation (MSLT, four choice reaction time test) was repeated. Alertness-related problems while driving were evaluated using the same questionnaire that had been used 1 year previously. Again, a second questionnaire, this time dealing with symptoms of sleep apnoea, quality of life, sleep habits, and usage of and experiences with nCPAP, was also completed.

Compliance was assessed both by questionnaire and, where available, by reading the inbuilt timing-meters of each nCPAP machine.

\section{Questionnaires}

The questionnaire dealing with alertness-related problems while driving, which was applied prior to treatment, consisted of the following items: 1) Do you possess a driver' s license? (yes/no); 2) How many kilometers do you drive per year? 3) As a driver of a motor vehicle, are you ever very tired? (5-point rating never, seldom, sometimes, frequently, always); 4) As a driver of a motor vehicle, do you ever doze off or startle and if so, how often per month? 5) As a driver of a motor vehicle, were you involved in accidents in the past five years? (yes/no; if yes, total number of accidents and number of accidents due to falling asleep, fatigue, or inability to maintain a sufficient level of concentration).

The same questionnaire (except for accident rate, where "in the past five years" was substituted by "in the past year") was used after 1 year of treatment of sleepdisordered breathing with nCPAP.

The two other questionnaires (33 and 51 items) that were given to the patients before treatment and at follow-up as part of the regular practice in the sleep laboratory, contained two items which will be presented in this paper: 1) How is your sense of smell? (5-point rating very good, good, average, bad, very bad); and 2) How is your vision? (5-point rating very good, good, average, bad, very bad). These items were not expected to change with treatment, and served as "dummy" items to indicate whether patient's tend to "tell us what we would like to hear". Significant positive changes in these items would clearly limit the meaningfulness of all questionnaire data.

\section{Vigilance test}

This test is very simple. It is intended to show impairments in long-term concentration as a correlate of reduced vigilance, and has been shown to be sensitive to treatment of SRBD with nCPAP (shorter reaction times with treatment) in previous studies [24, 25]. Four diamonds are visible on a screen, one (place randomized) disappears after a randomized interstimulus interval [20-50 s]. After the patient presses the corresponding button on a mini-keyboard, the diamond reappears. If the reaction time exceeds $10 \mathrm{~s}$, the diamond reappears and a beep loud enough to awaken a sleeping patient sounds. Mean reaction time and number of missed events are measured throughout the test.

\section{Data analysis}

MESAM recordings were visually evaluated by experienced technicians, and the Respiratory Disturbance Index for each patient was determined [22], in order to 
define the necessity of treatment with nCPAP in conjunction with other clinical features (e.g. disturbances of heart rhythm, subjective sleepiness).

Polysomnographic recordings were performed and evaluated according to the criteria published by the German Sleep Society [26]. Sleep stages were classified in $30 \mathrm{~s}$ periods, according to the criteria of RECHTSCHAFFEN and KALES [27]. Apnoea was defined as the cessation of oronasal airflow for at least $10 \mathrm{~s}$. Hypopnoea was defined as a reduction of oronasal airflow by at least $50 \%$ lasting for $10 \mathrm{~s}$ or more, accompanied by a decrease in $\mathrm{Sa}, \mathrm{O}_{2}$ of at least $4 \%$ of the preceding stable $\mathrm{Sa}_{\mathrm{a}} \mathrm{O}_{2}$. The number of apnoeas and hypopnoeas per hour of sleep time was calculated and reported as the apnoea hyponoea index (AHI). For each apnoea or hypopnoea, the $\mathrm{Sa}_{2} \mathrm{O}_{2}$ level just before the beginning of the desaturation and the $\mathrm{Sa}, \mathrm{O}_{2}$ nadir were recorded.

\section{Questionnaires}

Patients reported the weekly or monthly frequency of "dozing-off" or "startling" while driving a car. Based on their reported annual distance driven, the frequency of these events per 1,000 km was calculated, and mean values before and with 1 year of nCPAP therapy were computed. "Severe fatigue" while driving was rated on a 5 point scale (never, seldom, sometimes, frequently, always). Median ratings before and with 1 year of nCPAP therapy were calculated. Reported accidents in the 5 yrs before treatment and the year between the beginning of treatment and follow-up were standardized, and expressed as accident rate per $100,000 \mathrm{~km}$ based on the patients reported annual driving distance. These individual accident rates were averaged to result in pre- and posttreatment group accident rates.

Median ratings of the dummy items (sense of smell, vision; 5-point rating scale) before and with 1 year of nCPAP therapy were determined.

\section{Vigilance test}

Results of the vigilance test were expressed as average reaction time and average number of missed events (reaction time $>10 \mathrm{~s}$ ) over the total 80 min testing period for pretreatment and follow-up evaluations.

\section{Multiple sleep latency test}

Latencies to Stage 1 sleep were determined by visual analysis of recording periods with a duration of $30 \mathrm{~s}$. Sleep onset was, according to standard criteria [27], defined as the beginning of the first period of Stage 1 sleep. Individual mean sleep latencies of all sleep latency tests were averaged. Mean group sleep latencies before and after 1 year of treatment were calculated.

\section{Statistical analysis}

The main issue that this study addressed was to determine whether treatment with nCPAP for 1 year reduced motor vehicle accident rates in patients with SRBD. We considered that this would be true if the accident rate was reduced statistically significantly following the introduction of therapy. Data from a previous study [6] were used to perform a sample size determination [28], with the aim of ensuring the statistical demonstrability of a change from an average accident rate of $0.6 \pm 1( \pm \mathrm{SD})$ (estimate for pretreatment, derived from patients with most severe apnoea in the preceding study) to an accident rate of $0.15 \pm 0.35$ (estimate for posttreatment, derived from patients without sleep apnoea in preceding study). Results were considered significant when a p-value less than 0.05 was achieved (two-tailed testing). A $\beta$-error of 0.15 was considered to be acceptable.

This resulted in a required sample size of 55. In order to have at least 55 patients for pre/post comparisons, and figuring noncompliance with the protocol of the study to be less than $25 \%$, it was decided to enrol at least 75 patients.

The statistical analysis of all other dependent variables was performed exploratively and would not have been performed if significant changes in accident rate had not occurred. These variables were considered to mirror reported (sleeping spells, fatigue) and objective (vigilance, daytime sleepiness) accident-favouring factors. According to the nature of the dependent variables, parametric (Student's t-test) or nonparametric (Wilcoxon Signed Ranks Test) one sample tests were performed.

\section{Results}

Seventy eight patients were initially enrolled in the study with a mean ( \pm SEM) age of $48 \pm 1.1$ yrs. Mean body mass index (BMI) was $31 \pm 0.6 \mathrm{~kg} \cdot \mathrm{m}^{-2}$. Mean pretreatment AHI (apnoeas+hypopnoeas $\cdot \mathrm{h}^{-1}$ sleep) was $34.2 \pm 3.1$ events $\cdot \mathrm{h}^{-1}$.

Nasal CPAP was titrated to an average pressure of $8.9 \pm 0.26 \mathrm{cmH}_{2} \mathrm{O}$. The mean annual distance driven by the group was $29,606 \pm 2,367 \mathrm{~km} \cdot \mathrm{yr}^{-1}$.

In 19 patients, the study could not be completed. Twelve patients who were using nCPAP regularly attended the follow-up in the out-patient department but did not wish to undergo another sleep study or objective testing of sleepiness. Three patients discontinued nCPAP, and a further four patients could not be relocated. Thus, 7 out of 78 patients must be assumed to have discontinued treatment, yielding a noncompliance rate of $9 \%$.

Timing meter data were available for 44 out of the 71 patients continuing nCPAP treatment. In these 44 patients, objective average nightly use was $6.1 \pm 0.16 \mathrm{~h}$, with a minimum of $4 \mathrm{~h}$ and a maximum of $8.3 \mathrm{~h}$ nightly use. Objective nCPAP use of patients who finished the study $(6.13 \pm 0.17 \mathrm{~h} ; \mathrm{n}=37)$ did not differ from the nightly use of patients who did not finish the study $(5.94 \pm 0.47 \mathrm{~h} ; \mathrm{n}=7)$. Average reported nightly use of nCPAP of the 71 patients who continued using nCPAP after 1 year was $7.17 \pm 0.13 \mathrm{~h}$. The shortest reported nightly use was $5 \mathrm{~h}$, the longest $10 \mathrm{~h}$. Reported nightly use by the 44 patients whose machine included a counter $(7.2 \pm 0.16 \mathrm{~h})$ did not differ significantly from the reported use of those without counter $(7.11 \pm 0.2 \mathrm{~h})$. There was no significant difference of reported nightly use in the 12 patients who did not fulfil the study protocol $(7 \pm 0.4$ h), compared to the 59 who did complete all follow-up evaluations $(7.2 \pm 0.13 \mathrm{~h})$.

In these remaining 59 study patients, mean age was $49 \pm 1 \mathrm{yrs}$, mean BMI was $31.7 \pm 0.73 \mathrm{~kg} \cdot \mathrm{m}^{-2}$, and the average pretreatment AHI was $38.9 \pm 3.4$ events $\cdot h^{-1}$. Annual 
a)

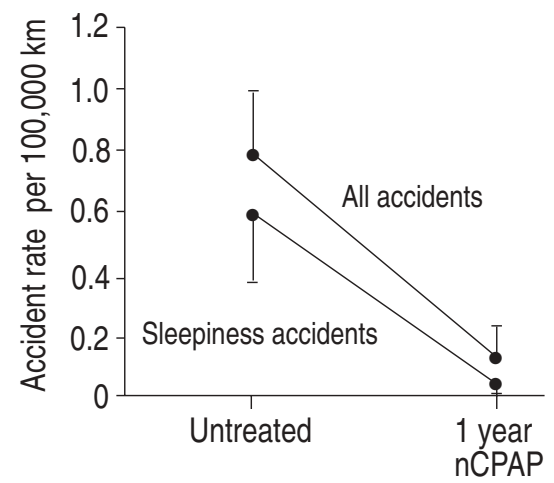

b)

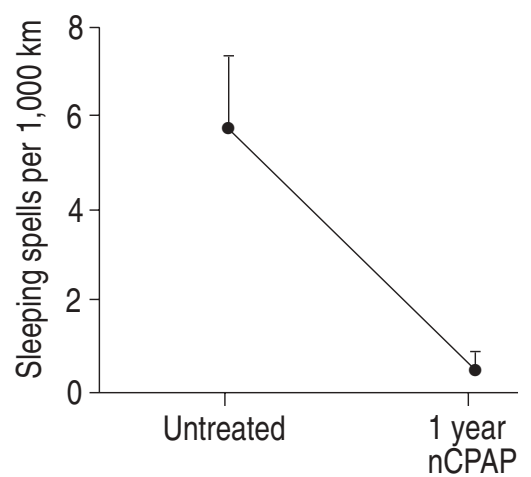

c)

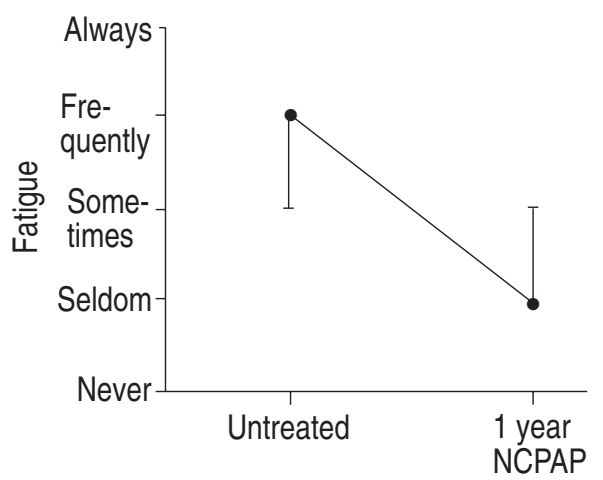

Fig. 1. - Alertness-related problems while driving. a) Accident rate per 100,000 km (+SEM) before and after 1 year of nCPAP treatment, and sleepiness accident rate per $100,000 \mathrm{~km}$ (-SEM) (accidents rated by patients to be caused by falling asleep, fatigue or inability to maintain a sufficient level of concentration) before and after 1 year of nCPAP treatment. b) Mean frequency of dozing off or startling per $1,000 \mathrm{~km}(+\mathrm{SEM})$ before and after 1 year of nCPAP treatment. c) Median rating of severe fatigue/decreases in alertness whilst driving, before and after 1 year of nCPAP treatment \pm 1 quartile (untreated: $75 \%$ quartile = median; 1 year nCPAP: $25 \%$ quartile = median). nCPAP: nasal continuous positive airway pressure.

distance driven was $29,860 \pm 2,886 \mathrm{~km} \cdot \mathrm{yr}^{-1}$. In the initial questionnaire, 29 of these patients $(44 \%)$ reported 40 accidents in the preceding 5 yrs, of which 27 were considered to be caused by falling asleep, fatigue, or inability to maintain a sufficient level of concentration. None of these variables (age, BMI, distance driven, AHI, reported accident rate) statistically differentiated the final 59 patients from those 19 patients who did not complete the study.

After 1 year of nCPAP, mean BMI was $31.6 \pm 0.7 \mathrm{~kg} \cdot \mathrm{m}^{-2}$ and the mean AHI was reduced to $3.1 \pm 1.3$ events $\cdot \mathrm{h}^{-1}$. Annual driving distance remained virtually unchanged at $30,859 \pm 3,340 \mathrm{~km} \cdot \mathrm{yr}^{-1}$.

\section{Questionnaire results: reported accidents and driving behaviour}

Both items intended to test the patients tendency towards socially desired (positive) responses showed no improvement with treatment. Sense of smell was rated to be "good" before and with treatment, vision was rated as "good" before treatment and "average" one year later (medians).

Before treatment, 0.8 accidents per $100,000 \mathrm{~km}$ were reported. With nCPAP, 0.15 accidents per $100,000 \mathrm{~km}$ occurred $(\mathrm{p}<0.01)$. A similar reduction was evident when the main cause of the accident was taken into account. Accidents rated to be caused by falling asleep, fatigue, or inability to maintain a sufficient level of concentration were reduced from 0.6 accidents per $100,000 \mathrm{~km}$ to 0.06 accidents per $100,000 \mathrm{~km}(\mathrm{p}<0.05)$ (fig. 1a).

The frequency of "dozing-off" or "startling" whilst driving was reduced from an average of 5.8 per $1,000 \mathrm{~km}$ to 0.58 per $1,000 \mathrm{~km}(\mathrm{p}<0.01)$ (fig. $1 \mathrm{~b})$. Median rating of "severe fatigue" while driving before initiation of nCPAP treatment was "frequently" and was reduced to "seldom" after 1 year of treatment $(\mathrm{p}<0.001)$ (fig. 1c).

\section{MSLT}

Mean group sleep latencies of all MSLTs increased from $12.0 \pm 0.67 \mathrm{~min}$ (pretreatment) to $16.8 \pm 0.71 \mathrm{~min}$ with 1 year of nCPAP treatment $(\mathrm{p}<0.05)$.

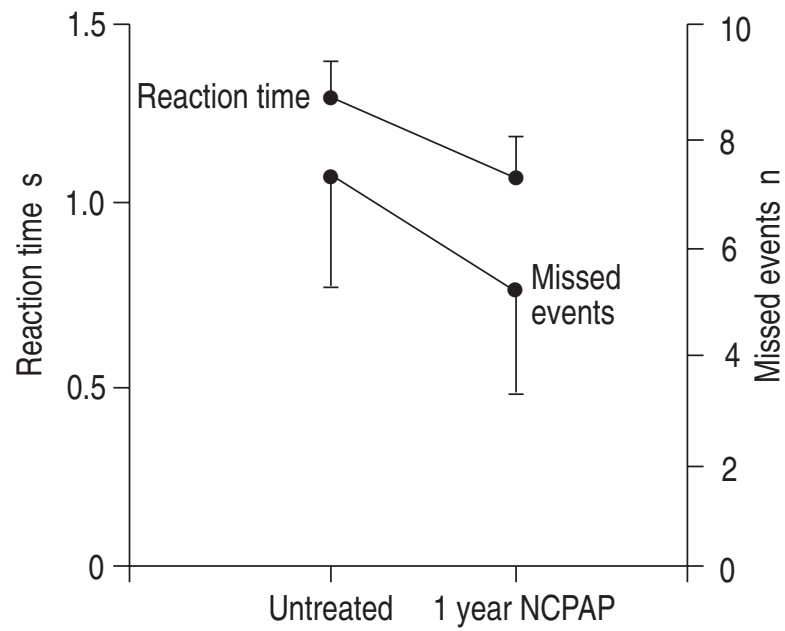

Fig. 2. - Mean reaction time and mean number of missed events before and after 1 year of nasal continuous positive airway pressure (nCPAP).

\section{Vigilance test}

Short reaction times and a low number of missed events over the course of the four choice reaction time test are indicative of an ability to sustain attentiveness under conditions of extreme monotony.

Mean reaction time shortened by $0.22 \mathrm{~s}$ from 1.3 to $1.08 \mathrm{~s}(\mathrm{p}<0.05)$. The mean number of missed events changed from 7.2 to 5.1 with treatment; however, this reduction failed to reach statistical significance $(\mathrm{p}=0.25)$ (fig. 2).

\section{Discussion}

Although it has been shown that accident risk is increased in untreated patients with sleep-disordered breathing [6-14], there is, to our knowledge, only one report (of 14 patients) providing information about the effect of nCPAP on accidents [19]. The results of this study in 59 patients indicate that treatment of SRBD with nCPAP for 12 months was related to a profound decrease of accident rate, which was accompanied by improved vigilance and reduced sleepiness. 
A total of 19 patients failed to complete the 1 year study follow-up. The 12 patients withdrawing their consent did not report any accidents in the preceding year, were clinically well-treated by nCPAP, and reported good subjective success of the treatment. Nevertheless, perhaps because they felt well-treated, they did not wish to repeat the additional daytime testing procedures.

The noncompliance rate of $9 \%$ (7 out of 78) reported here is relatively low when compared to other studies $[29,30]$, in which higher rates of noncompliance have been reported, up to $36 \%$ in one study [31]. The high compliance rate found in the present study might be due to the selection of patients, who differed from "average" patients in several ways. Firstly, reported sleepiness was an inclusion criterion for this study, thus patients with a substantial potential subjective benefit from nCPAP were selected. Furthermore, it seems reasonable that patients who agree to participate in an elaborate and time-consuming study protocol could be assumed to show a higher than average compliance with nCPAP. Another possible cause might be the diligent and thorough method of pressure determination and the continuing contact and liaison with the sleep laboratory and the referring physician over the study period.

The use of subjective data regarding motor vehicle accidents is currently unavoidable in Germany, due to the legal restrictions on the protection of personal data. Thus, a potential bias in the patients reports cannot be ruled out completely.

We tried to reduce this risk by encouraging all patients to also report negative experiences with treatment, as these will be most valuable in the long-term in improving the therapy of SRBD. The stability of the dummy items and the numerous shortcomings of nCPAP reported (noisy, machines too big, masks need improvement, etc) suggest that there was no strong bias towards positive responses in this study. Of course, this does not exclude a bias resulting from positive effects of variables that are not directly related to treatment by nCPAP, such as education about sleepiness and driving, and knowledge about participation in a study.

The relative contribution of treatment itself and other effects, such as regression to the mean, increasing the patients' awareness about the hazards of driving while being sleepy and knowledge about participation in a study have to remain uncertain, as no control group of comparably affected patients was left untreated and uninformed about the hazards of sleepiness while driving. Taking all these additional factors into account, we still believe a relevant proportion of the patients' reduction in accident rate to be due to the nCPAP treatment, and that the amount of the reduction in accident rate is meaningful in itself, even though specific contributions of factors involved in this improvement cannot be isolated in this study.

Although including an untreated control group is without doubt desirable from a scientist's point of view, it could not be done in this study, as not treating a sleepy patient with sleep-related breathing disorders and not even giving information about the potential hazards of sleepiness and fatigue while driving (the proper way of handling a control group in a prospective study) would be regarded as an offence in Germany.

The questionnaire dealing with alertness related problems while driving was applied in several preceding studies [6, 32-34], which clearly showed lower rates of reported accidents and less vigilance-related problems while driving in healthy controls as compared to patients with SRBD.

The accident rate of untreated patients reported in this study of 0.8 accidents per $100,000 \mathrm{~km}$ is higher than the German average of 0.42 accidents per $100,000 \mathrm{~km}$ [35]. Because patients might fear negative consequences from admitting to motor vehicle accidents, we feel these numbers are likely to be a lower estimate of the patients "true" accident rate. Accordingly, patients' accident rate in the first year after initiation of nCPAP $(0.15)$ is lower than the overall average.

In addition to the reduced number of reported traffic accidents, potential SRBD-related problems, such as sleeping spells and severe fatigue while driving, were also greatly reduced with nCPAP therapy.

Pretreatment MSLT sleep latencies (12 min) were in the lower normal range [23], indicating only a slightly increased overall level of daytime sleepiness in the patients studied. As reported previously [36, 37], these results indicate a poor agreement between reported sleepiness and objective sleepiness as measured by the MSLT.

Even though patients were not severely impaired, according to MSLT standard criteria [23], a significant prolongation (+4 min $45 \mathrm{~s})$ of sleep latencies occurred after 1 year of nCPAP, indicating a lower level of daytime sleepiness in treated patients. Furthermore, following 1 year of nCPAP treatment, a slight (but significant) reduction of reaction times in the $80 \mathrm{~min}$ vigilance task was measured. The results of these objective tests are viewed by us as evidence for an improved ability to sustain attentiveness under conditions of extreme monotony.

According to the results obtained by Minemura et al. [19] in a small sample $(n=14)$, this study indicates that treatment of sleep-disordered breathing by nCPAP does indeed reduce patients' proneness to accidents, even though the relative contribution of factors such as regression to the mean, awareness about the risk of sleepiness while driving and knowledge about participation in a study, cannot be isolated due to the lack of a control group. Nevertheless, objective data on changes in vigilance and sleepiness, as well as reported reductions of alertness-related problems while driving, are in agreement with the observed reduction in accident rate.

In professional drivers and other persons with potentially dangerous occupations suffering from sleep-disordered breathing with impaired alertness, continuing success of treatment should be documented on a yearly basis, by means of recording sleep-disordered breathing and with objective tests of daytime vigilance and daytime sleepiness.

Taking the high risk and costs of sleepiness-related accidents into account $[3,5]$, and in view of the likely chance of effective treatment of sleep-disordered breathing in most patients [17], efforts to identify and treat these patients have to be reinforced. There is increasing evidence for a positive cost-benefit relationship of such efforts, when costs of health care are compared to economic benefits through possible accident prevention [5]. This relationship might be even more positive if the likely decrease in the probability of early retirement 
and the possible reduction of accidents in the workplace with effective treatment of sleep-related breathing disorders are taken into account.

\section{References}

1. Dinges DF. An overview of sleepiness and accidents. J Sleep Res 1995; 4 (Suppl. 2): 4-14.

2. Horne JA, Reyner LA. Driver sleepiness. J Sleep Res 1995; 4 (Suppl. 2): 23-29.

3. Langwieder K, Sporner A, Hell W. Struktur der Unfälle mit Getöteten auf Autobahnen im Freistaat Bayern im Jahr 1991. München, HUK-Verband, Büro für KfzTechnik, 1994.

4. Mitler MM, Carskadon MA, Czeisler CA, Dement WC, Dinges DF, Graeber RC. Catastrophes, sleep, and public policy: consensus report. Sleep 1988; 11(1): 100-109.

5. Leger D. Cost of sleep-related accidents. Sleep 1994; 17 (1): 84-93.

6. Cassel W, Ploch T. Schlafbezogene Atmungsstörungen: Unfallgefahr als psychosozialer Risikofaktor. In: Hecht K, Engfer A, Peter JH, Poppei M, (eds). Schlaf, Gesundheit, Leistungsfähigkeit. Berlin, Heidelberg, New York, Springer, 1993; pp. 233-242.

7. Findley L, Unverzagt ME, Guchu R, Fabrizio M, Buckner $\mathrm{J}$, Suratt $\mathrm{P}$. Vigilance and automobile accidents in patients with sleep apnea or narcolepsy. Chest 1995; 108 (3): 619-624.

8. Findley LJ, Unverzagt ME, Surrat PM. Automobile accidents involving patients with obstructive sleep apnea. Am Rev Respir Dis 1988; 138: 337-340.

9. Findley LJ, Weiss WJ, Jabour ER. Drivers with untreated sleep apnea: a cause of death and serious injury. Arch Intern Med 1991; 151: 1451-1452.

10. George C, Nickerson P, Hanly P, Millar T, Kryger M. Sleep apnea patients have more automobile accidents (Letter). Lancet 1987; i: 447.

11. Haraldsson PO, Carenfelt C, Diderichsen F, Nygren A, Tingvall C. Clinical symptoms of sleep apnea syndrome and automobile accidents. ORL 1990; 52 (1): 57-62.

12. Haraldsson PO, Carenfelt C, Tingvall C. Sleep apnea syndrome symptoms and automobile driving in a general population. J Clin Epidemiol 1992; 45: 821-825.

13. American Thoracic Society. Sleep apnea, sleepiness, and driving risk. Am J Respir Crit Care Med 1994; 150: 1463-1473.

14. Findley LJ, Fabrizio MJ, Knight H, Norcross BB, Laforte AJ, Suratt PM. Driving simulator performance in patients with obstructive sleep apnea. Am Rev Respir Dis 1989; 140 (2): 529-530.

15. Haraldsson PO, Carenfelt C, Persson HE, Sachs C, Törnros J. Simulated long-term driving performance before and after uvulopalatopharyngoplasty. ORL 1991; 53: 106-110.

16. Sullivan CE, Issa FG, Berthon-Jones M, Eves L. Reversal of obstructive sleep apnea by continuous positive airway pressure applied through the nares. Lancet 1981; i: 862-865.

17. Becker H, Peter JH, von Wichert P. Nasal continuous positive airway pressure. Eur Respir Rev 1992; 10: 400-408.

18. He JH, Kryger MH, Zorick FJ, Conway W, Roth T. Mortality and apnea index in obstructive sleep apnea: experience in 385 male patients. Chest 1988; 94: 9-14.

19. Minemura H, Akashiba T, Yamamoto H, et al. Traffic accidents in obstructive sleep apnea patients and effect of nasal CPAP treatment. Nippon Kyobo Shikkan Gakkai Zasshi 1993; 31 (9): 1103-1108.
20. Penzel T, Althaus W, Meinzer K, Peter JH, von Wichert P. A device for ambulatory heart rate, oxygen saturation and snoring recording. Proceedings of the Annual International Conference of the IEEE Engineering in Medicine and Biology Society. 1991; 13: 1616-1617.

21. Stoohs R, Guilleminault C. MESAM 4: an ambulatory device for the detection of patients at risk for obstructive sleep apnea syndrome (OSAS). Chest 1992; 101: 1221-1227.

22. Roos M, Althaus W, Rhiel C, Penzel T, Peter JH, von Wichert P. Vergleichender Einsatz von MESAM IV und Polysomnographie bei schlafbezogenen Atmungsstörungen (SBAS). Prax Klin Pneumol 1993; 47: 112-118.

23. Carscadon MA. Guidelines for the Multiple Sleep Latency Test (MSLT): A standard measure of sleepiness. Sleep 1986; 9 (4): 518-524.

24. Cassel W, Vogel M, Moog R, Ploch T, Peter JH, von Wichert P. 24 hours spontaneous sleep, multiple sleep latencies, reaction time and subjective sleepiness in patients with obstructive sleep apnea. Sleep Res 1991; 20A: 527.

25. Schwarzenberger-Kesper F, Becker H, Penzel T, Peter JH, Weber K, von Wichert P. Die exzessive Einschlafneigung am Tage (EDS) beim Apnoe-Patienten: Diagnostische Bedeutung und Objektivierung mittels Vigilanztest und synchroner EEG-Registrierung am Tage. Prax Klin Pneumol 1987; 41: 401-405.

26. Penzel T, Hajak G, Hoffmann RM, et al. Empfehlungen zur Durchführung und Auswertung polygraphischer Ableitungen im diagnostischen Schlaflabor. Z EEG EMG 1993; 24: 65-70.

27. Rechtschaffen A, Kales A. A manual of standardized terminology, techniques and scoring system for sleep stages of human subjects. Public Health Service Publication 204, US Government Printing Office, Washington DC, 1968.

28. Hays WL. Statistics. 4th Edn. Fort Worth, Holt, Rinehart and Winston, 1988.

29. Fleury B, Rakotonanahary D, Tehindrazanarivelo AD, Hatsser-Hauw C, Lebeau B. Long-term compliance to continuous positive airway pressure therapy (nCPAP) set-up during a split-night polysomnography. Sleep 1994; 17: $512-515$.

30. Sanders MH, Gruendl CA, Rogers RM. Patient compliance with nasal CPAP therapy for sleep apnea. Chest 1986; 90: 330-333.

31. Rolfe I, Olson LG, Saunders NA. Long-term acceptance of continuous positive airway pressure in obstructive sleep apnea. Am Rev Respir Dis 1991; 144: 1130-1133.

32. Cassel W. Sleep apnea and accidents: health risk for healthy people? In: Peter JH, Penzel T, Podszus T, von Wichert P, eds. Sleep and Health Risk. Berlin, Heidelberg, New York, Springer, 1990; pp. 279-285.

33. Cassel W, Ploch T, Peter JH, von Wichert P. Disordered breathing, sleep and driving. Eur Respir J 1990; 3 (Suppl. 10): $149 \mathrm{~s} / 438$.

34. Cassel W, Ploch T, Nees E, Fett I, Peter JH, von Wichert P. Patients with obstructive sleep apnea are sleepy while driving but drive nevertheless. Sleep Res 1990; 19: 116.

35. Statistisches Bundesamt Wiesbaden. Fachserie 8 Verkehr, Reihe 7 Verkehrsunfälle. Stuttgart Mainz, Kohlhammer, 1992.

36. Johnson LC, Spinweber CL, Gomez SA, Matteson LT. Daytime sleepiness, performance, mood, nocturnal sleep: the effect of benzodiazepine and caffeine on their relationship. Sleep 1990; 13: 121-135.

37. Moldofsky H. Evaluation of daytime Sleepiness. Clin in Chest Med 1992; 13 (3): 417-425 